\title{
Article \\ BRAF Mutation Is Associated with Hyperplastic Polyp-Associated Gastric Cancer
}

\author{
Rina Fujiwara-Tani ${ }^{1}$, Ayaka Okamoto ${ }^{1}$, Hiroyuki Katsuragawa ${ }^{1}$, Hitoshi Ohmori ${ }^{1}$, Kiyomu Fujii ${ }^{1}$, Shiori Mori ${ }^{1}$, \\ Shingo Kishi ${ }^{1}$, Takamitsu Sasaki ${ }^{1}$, Chie Nakashima ${ }^{1}$, Isao Kawahara ${ }^{1}$, Yudai Hojo ${ }^{1}$, Yukiko Nishiguchi ${ }^{1}$, \\ Takuya Mori ${ }^{1}$, Takeshi Mizumoto ${ }^{2}$, Kenta Nagai ${ }^{2}$, Yi Luo ${ }^{3, *}$ and Hiroki Kuniyasu ${ }^{1, *}$ (D)
}

1 Department of Molecular Pathology, Nara Medical University, 840 Shijo-cho, Kashihara 634-8521, Nara, Japan; rina_fuji@naramed-u.ac.jp (R.F.-T.); dc117023@naramed-u.ac.jp (A.O.); mol.path.hk@gmail.com (H.K.); brahmus73@hotmail.com (H.O.); toto1999-dreamtheater2006-sms@nifty.com (K.F.); m.0310.s.h5@gmail.com (S.M.); nmu6429@yahoo.co.jp (S.K.); takamitu@fc4.so-net.ne.jp (T.S.); c-nakashima@naramed-u.ac.jp (C.N.); isao_kawahara@a011.broada.jp (I.K.); yudaihojo@outlook.com (Y.H.); yukko10219102@yahoo.co.jp (Y.N.); pt_mori_t@yahoo.co.jp (T.M.)

2 Miyoshi Central Hospital, 10531 Higashi-Sakaya-cho, Miyoshi 728-8502, Japan; T.Mizumoto@city.miyoshi.hiroshima.jp (T.M.); K.Nagai@city.miyoshi.hiroshima.jp (K.N.)

3 Key Laboratory of Neuroregeneration of Jiangsu and Ministry of Education, Co-Innovation Center of Neuroregeneration, Nantong University, Nantong 226001, China

* Correspondence: lynantong@hotmail.com (Y.L.); cooninh@zb4.so-net.ne.jp (H.K.); Tel.: +86-0513-85051805 (Y.L.); +81-744-22-3051 (H.K.); Fax: +81-744-25-7308 (H.K.)

check for updates

Citation: Fujiwara-Tani, R.; Okamoto, A.; Katsuragawa, H.; Ohmori, H.; Fujii, K.; Mori, S.; Kishi, S.; Sasaki, T.; Nakashima, C.; Kawahara, I.; et al. BRAF Mutation Is Associated with Hyperplastic Polyp-Associated Gastric Cancer. Int. J. Mol. Sci. 2021, 22, 12724. https:// doi.org/10.3390/ijms222312724

Academic Editor: Peter J.K. Kuppen

Received: 29 October 2021

Accepted: 22 November 2021

Published: 25 November 2021

Publisher's Note: MDPI stays neutral with regard to jurisdictional claims in published maps and institutional affiliations.

Copyright: (C) 2021 by the authors. Licensee MDPI, Basel, Switzerland. This article is an open access article distributed under the terms and conditions of the Creative Commons Attribution (CC BY) license (https:/ / creativecommons.org/licenses/by/ $4.0 /)$.
Abstract: Gastric hyperplastic polyps (GHP) are frequently found to be benign polyps and have been considered to have a low carcinogenic potential. The characteristics of the hyperplastic polypassociated gastric cancer (HPAGC) remain unclear. Therefore, we analyzed samples from 102 GHP patients and identified 20 low-grade atypical GHPs (19.6\%), 7 high-grade atypical GHPs (6.9\%), and 5 intramucosal cancer samples $(4.9 \%)$. GHP atypia was more common in the elderly and increased with increasing polyp size. In particular, polyps larger than $1 \mathrm{~cm}$ were associated with a higher grade and cancer. Furthermore, mucus production decreased with increasing atypia. Although no correlation was found between atypia and Helicobacter pylori infection or intestinal metaplasia, enhanced proliferative ability (Ki-67) did correlate with atypia, as did nuclear 8-hydroxy2'-deoxyguanosine levels. Interestingly, 4-hydroxynonenal levels in granulation tissue and the area ratio of granulation tissue within polyps also correlated with GHP atypia. In five cases of HPAGC, three cases exhibited caudal type homeobox transcription factor (CDX2)-positive cells and a mixed mucin phenotype, which is considered to be related to H. pylori infection. By contrast, two cases were CDX2 negative, with a gastric mucin phenotype, and $H$. pylori infection was not observed in the tumor or the surrounding mucosa. In these cases, a v-raf murine sarcoma viral oncogene homolog B1 (BRAF) mutation (V600E) was detected. All cancer samples showed high stemness and p53 protein accumulation, but no KRAS mutations. The molecular and phenotypic characteristics of the cases characterized by BRAF mutations may represent a novel subtype of HPAGC, reflecting a conserved pathway to oncogenesis that does not involve $H$. pylori infection. These findings are worthy of further investigation in a large-scale study with a substantial cohort of HPAGC patients to establish their clinical significance.

Keywords: gastric hyperplastic polyp; gastric cancer; BRAF mutation; H. pylori; oxidative stress

\section{Introduction}

Gastric cancer is currently the third most common cause of cancer-related death in Japan [1]. Inflammation is a key feature of gastric cancer, and oxidative stress due to chronic inflammation caused by Helicobacter pylori plays an important role in the carcinogenesis of gastric cancer [2]. Chronic atrophic gastritis and intestinal epithelialization are known to be precancerous conditions caused by H. pylori [3]. In our previous study, we showed 
that the activation of oxidative stress and v-akt murine thymoma viral oncogene homolog (AKT) increased substantially in each of the following categories of chronic gastritis: chronic gastritis without $H$. pylori, chronic active gastritis with $H$. pylori, chronic metaplastic gastritis without $H$. pylori, and chronic gastritis with atypia without $H$. pylori [4]. This is due to the shortening of telomeres and activation of telomerase reverse transcriptase, which are associated with repeated mucosal regeneration due to chronic inflammation [5] and are thought to lead to hyperplasia of gastric mucosal stem cells [6,7].

Gastric hyperplastic polyps (GHPs) are the most common polyps encountered in the stomach (along with fundic gland polyps) and are detected in 1.9\% of 110,000 patients subjected to gastroscopy [8]. Patients with GHPs are usually asymptomatic; however, some may present with dyspepsia, heartburn, abdominal pain, anemia, or upper gastrointestinal bleeding [9]. GHPs are thought to occur during the repair of damaged mucosa [9]. Malignant transformation of GHP is very infrequent, with an incidence of $0.3-3 \%[10,11]$. In previous research, hyperplastic polyp-associated gastric cancer (HPAGC) is derived from dysplastic foci in GHP [12,13]. However, the histological and molecular pathological markers for its definitive diagnosis have not been established.

Recently, genome and proteome analyses of gastric cancer have been performed as part of The Cancer Genome Atlas (TCGA) project, and it has been established that gastric cancer can be classified into four molecular subtypes [14]: (1) Epstein-Barr virus, (2) microsatellite instability, (3) genomically stable, and (4) chromosomal instability subtypes. Such comprehensive molecular pathological analysis provides a better understanding of the development of gastric cancer and enables the extraction of molecular targets that lead to drug discovery. However, due to the scarcity of cases, the details of HPAGC have not been completely clarified. Doing so may improve our understanding of this specific type of gastric cancer and provide insight as to whether different treatment approaches are required to deal with this cancer. In this study, we investigated the characteristics of early HPAGC and analyzed its underlying pathogenic mechanisms and molecular abnormalities.

\section{Results}

\subsection{GHP Atypia and the Associated Clinical and Morphological Characteristics}

First, we classified GHP atypia into four categories: non-atypical GHP with no nuclear and no structural atypia with abundant mucus production; low-grade atypia with mild to moderate nuclear swelling; high-grade atypia with moderate to prominent nuclear swelling, pseudostratified nuclei, and decreased periodic acid-Schiff (PAS)-positive mucus production; and HPAGC with the cellular characteristics of high-grade atypia as well as structural atypia and/or invasion (Figure 1).

Using this categorization process to sort the samples from $102 \mathrm{GHP}$ patients, we identified 20 GHP samples as low-grade atypia, 7 GHP samples as high-grade atypia, and 5 GHP samples as HPAGC (Table 1). Atypical GPHs were more common in the elderly and the incidence increased with an increase in polyp size. In particular, polyps larger than $1 \mathrm{~cm}$ were associated with a higher grade and cancer. In addition, mucus production decreased with increasing atypia. By contrast, no correlation was observed between atypia and $H$. pylori infection or intestinal metaplasia. Immunohistochemistry for Ki-67 expression showed that enhanced proliferative activity also correlated with atypia (Figure 2). 


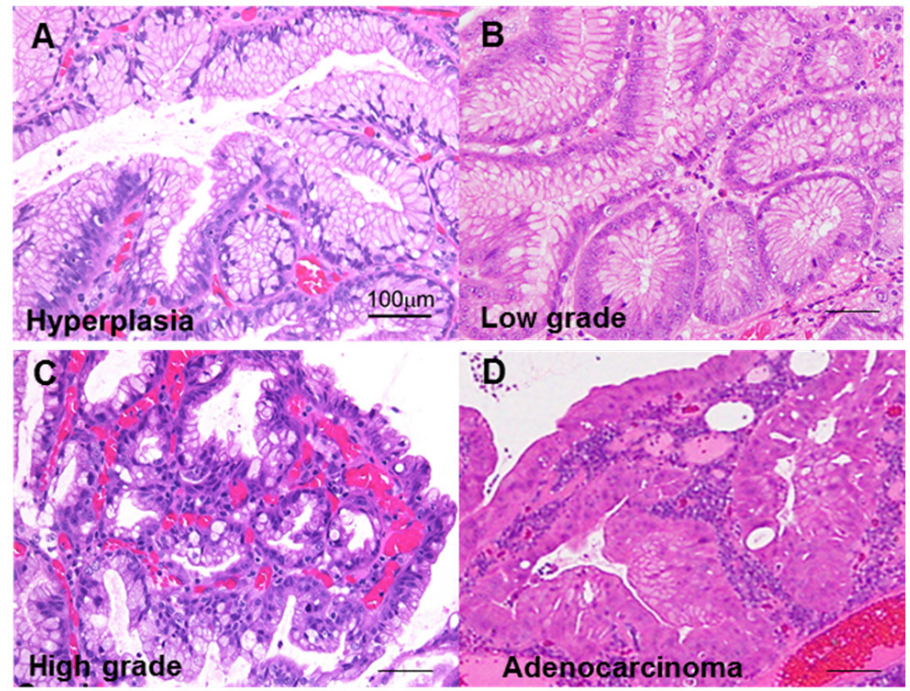

Figure 1. Atypia in hyperplastic polyps. (A) Hyperplastic polyp without atypia. (B) Low-grade atypia, notable mild nuclear swelling. (C) High-grade atypia, notable moderate to marked nuclear swelling, nuclear pseudostratification, and decrease in mucus production. (D) Carcinoma in hyperplastic polyp (well-differentiated tubular adenocarcinoma), marked nuclear swelling, nuclear pseudostratification, decrease in mucus production, and structural atypia. Scale bar, $100 \mu \mathrm{m}$.

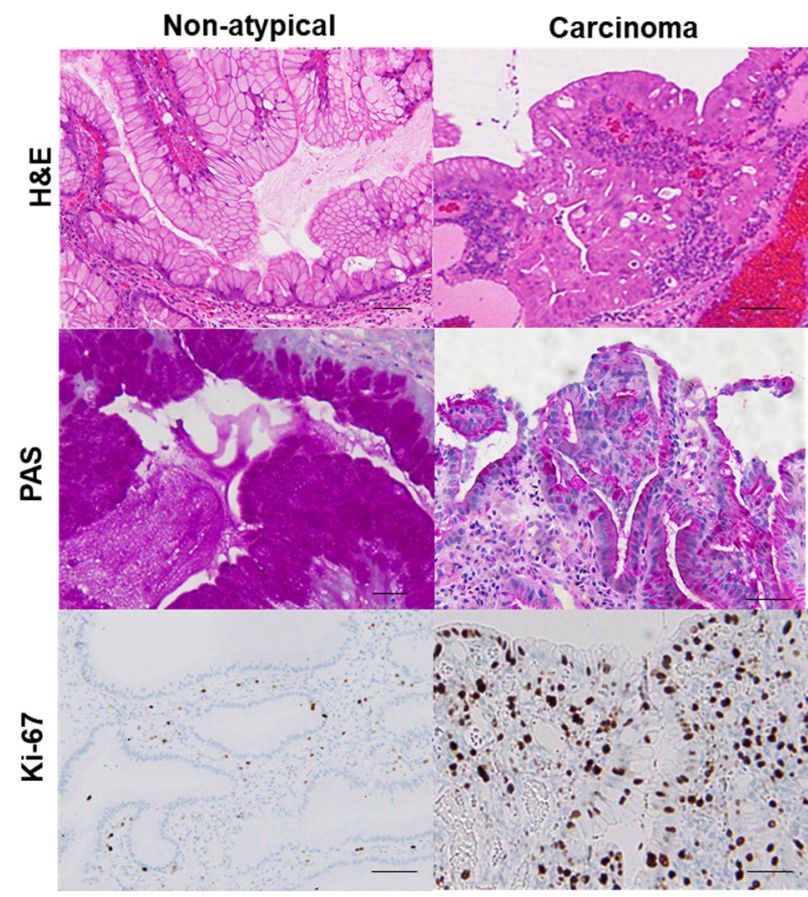

Figure 2. Comparison of mucus production and proliferative activity between a hyperplastic polyp and its carcinoma lesion.In the same hyperplastic polyp (case 2 in Table 3), non-atypical regions and the carcinoma lesion were compared. In the non-atypical region, PAS-positive mucus is abundant in most foveolar epithelial cells, whereas in the cancer lesion, PAS-positive mucus is only found in a few cells. Ki-67-positive cells were scattered in non-atypical regions with an index of $5 \%$. By contrast, in the cancer lesion, a high number of Ki-67-positive cells were evident, up to the surface layer of the glands (index of 92\%). H\&E, hematoxylin and eosin; PAS, periodic acid-Schiff. 
Table 1. Relationship between polyp atypia and H. pylori infection or proliferation.

\begin{tabular}{|c|c|c|c|c|c|}
\hline \multirow{2}{*}{ Parameter } & \multicolumn{4}{|c|}{ Polyp Atypia (1) } & \multirow{2}{*}{$p$-Value } \\
\hline & None & Low Grade & High Grade & Cancer & \\
\hline Number & 72 & 20 & 7 & 5 & \\
\hline Age (yrs) & $55 \pm 13$ & $60 \pm 11$ & $63 \pm 10$ & $70 \pm 5$ & 0.0191 \\
\hline Sex (male: female) & $22: 22$ & $12: 8$ & $4: 3$ & $3: 2$ & NS \\
\hline Size (mm) & $4.7 \pm 1.8$ & $6.9 \pm 1.7$ & $12.3 \pm 5.9$ & $14.6 \pm 5.4$ & $<0.0001$ \\
\hline \multicolumn{6}{|l|}{ H. pylori infection } \\
\hline Incidence $(\%)$ & 9 & 10 & 0 & 20 & NS \\
\hline Grade ${ }^{(2)}$ & $0.18 \pm 0.65$ & $0.10 \pm 0.31$ & 0 & $0.20 \pm 0.45$ & NS \\
\hline \multicolumn{6}{|l|}{ Intestinal metaplasia (2) } \\
\hline Incidence $(\%)$ & 32 & 55 & 29 & 40 & NS \\
\hline Grade $(2)$ & $0.5 \pm 0.88$ & $0.55 \pm 0.51$ & $0.29 \pm 0.49$ & $0.40 \pm 0.55$ & NS \\
\hline PAS staining $(3)$ & $2.0 \pm 0.1$ & $1.4 \pm 0.5$ & $0.8 \pm 0.3$ & $0.6 \pm 0.2$ & $<0.0001$ \\
\hline Ki-67 index $(\%)$ & $26 \pm 13$ & $52 \pm 23$ & $74 \pm 18$ & $86 \pm 10$ & $<0.0001$ \\
\hline
\end{tabular}

(1) Low grade, mild to moderate nuclear swelling; high grade, moderate to marked nuclear swelling, nuclear pseudostratification, and weakened mucus production; cancer, structural atypia, and/or invasion in addition to alterations similar to high-grade lesions. ${ }^{(2)}$ According to the updated Sydney classification [15], each grade (none, mild, moderate, and severe) was quantified as 0, 1, 2, and 3, respectively, and the results were statistically analyzed. ${ }^{(3)}$ PAS staining grades were classified according to the ratio of the area of the PAS-positive region in the cells: $0,0 \% ; 0.5,<10 \% ; 1,10-25 \% ; 2,25-50 \% ; 3,>50 \%$. ${ }^{(4)} \mathrm{Ki}-67$ staining was examined in 1000 epithelial cells and the frequency of positive nuclear staining was determined.

\subsection{GHP Atypia and Oxidative Stress}

Oxidative stress has a prominent influence on gastric carcinogenesis [2]. When we investigated the relationship between atypia and oxidative stress in GHPs (Figure 3, Table 2), we found a correlation between atypia and nuclear 8-hydroxy-2'-deoxyguanosine (8-OHdG) levels. Interestingly, 4-hydroxy nonenal (HNE) levels in the granulation tissue of GHPs also correlated with atypia, as did the area ratio of granulation tissue within the polyps. Nuclear 8-OHdG levels and granulation tissue area, or 4-HNE levels in granulation tissue, were all correlated (Pearson $r=0.6489$ and $0.7245, p<0.0001$ and $<0.0001$, respectively). These results suggest that granulation tissue may play a role in generating oxidative stress in GHP, and it may be a trigger for GHP carcinogenesis.

Table 2. Relationship between atypia and granulation tissue, oxidative stress, or stemness.

\begin{tabular}{|c|c|c|c|c|c|}
\hline \multirow{2}{*}{ Parameter } & \multicolumn{4}{|c|}{ Polyp Atypia ${ }^{(1)}$} & \multirow{2}{*}{$p$-Value } \\
\hline & None & Low Grade & High Grade & Cancer & \\
\hline Number & 44 & 20 & 7 & 5 & \\
\hline Granulation tissue (\%) (2) & $15 \pm 12$ & $48 \pm 23$ & $79 \pm 7$ & $87 \pm 7$ & $<0.0001$ \\
\hline Tumor 8-OHdG index (3) & $23 \pm 12$ & $52 \pm 18$ & $86 \pm 8$ & $94 \pm 5$ & $<0.0001$ \\
\hline 4-HNE (ng/g) ${ }^{(4)}$ & $0.5 \pm 0.1$ & $0.8 \pm 0.2$ & $1.8 \pm 0.3$ & $2.4 \pm 0.3$ & $<0.0001$ \\
\hline CDX2 incidence $(\%)$ & 25 & 50 & 29 & 40 & NS \\
\hline p53 incidence (\%) & 0 & 0 & 43 & 100 & 0.0075 \\
\hline NS index (\%) $(3)$ & $18 \pm 8$ & $53 \pm 17$ & $80 \pm 2$ & $84 \pm 6$ & $<0.0001$ \\
\hline
\end{tabular}

(1) Low grade, mild to moderate nuclear swelling; high grade, moderate to marked nuclear swelling, nuclear pseudostratification, and weakened mucus production; cancer, structural atypia, and/or invasion in addition to alterations similar to high-grade lesions. ${ }^{(2)}$ Evaluated according to the area occupied by the polyp: $0,<10 \% ; 1,10-50 \% ; 2,50-75 \% ; 3,>75 \%$. ${ }^{(3)}$ For assessment of these parameters, 1000 epithelial cells were examined, and the frequency of positive nuclear staining was determined. For p53 and CDX2, cases were judged as positive when the frequency of positive cells (index) was $10 \%$ or more. ${ }^{(4)} 4$-HNE levels in extracts from granulation tissues were measured using ELISA. 


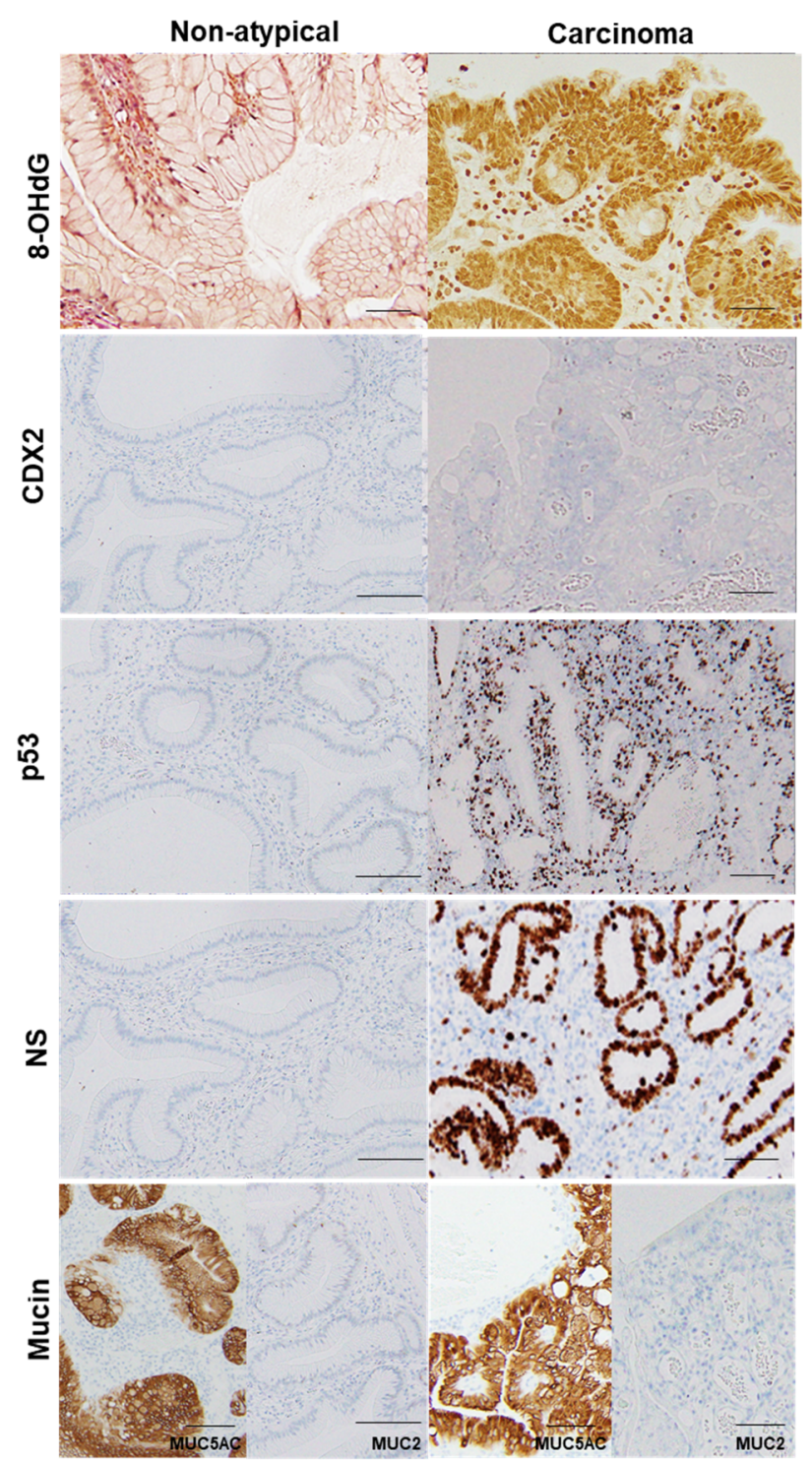

Figure 3. Comparison of oxidative stress, intestinal phenotype, and stemness between hyperplastic polyp and its carcinoma lesion.

In the same hyperplastic polyp (case 2 in Table 3), non-atypical regions and the carcinoma lesion were compared. 8-OHdG in the nuclei was scattered in non-atypical regions (index of $4 \%$ ), whereas the cancer lesions exhibited a substantial number of positive cells (index of 100\%). Both non-atypical regions and the cancer lesion showed no nuclear expression of CDX2. p53 nuclear accumulation was found in the cancer lesion (index of $72 \%)$, but not in non-atypical regions (index of $0 \%$ ). Nuclear staining for NS was only observed in a few cells in non-atypical regions (index of less than $1 \%$ ), whereas cancer lesions showed high labeling of NS (index of 87\%). 8-OHdG, 8-hydroxy-2'-deoxyguanosine; CDX2, caudal type homeobox transcription factor 2; NS, nucleostemin. 
Table 3. Adenocarcinoma cases in hyperplastic polyps.

\begin{tabular}{cccccc}
\hline & \multicolumn{5}{c}{ Case } \\
\cline { 2 - 6 } & $\mathbf{1}$ & $\mathbf{2}$ & $\mathbf{3}$ & $\mathbf{4}$ & $\mathbf{5}$ \\
\hline Polyp size (mm) & 11 & 8 & 20 & 20 & 14 \\
Cancer lesion (mm) & 2 & 4 & 3 & 3 & 2 \\
Histology (1) & tub1 & Pap + tub1 & tub1 & tub1 & Pap + tub1 \\
Invasion & In situ & In situ & Invasive & In situ & In situ \\
Mucin type (2) & Mixed & Gastric & Gastric & Mixed & Mixed \\
Ki-67 index (\%) & 94 & 92 & 85 & 70 & 90 \\
8-OHdG index (\%) $(3)$ & 100 & 100 & 92 & 88 & 90 \\
CDX2 index (\%) (3) & 24 & 0 & 0 & 5 & 80 \\
p53 index (\%) $(3)$ & 78 & 72 & 19 & 17 & 89 \\
NS index (\%) $(3)$ & 78 & 87 & 85 & 82 & 88 \\
KRAS G12D/G13D & $-/-$ & $-/-$ & $-/-$ & $-/-$ & $-/-$ \\
BRAF V600E & - & + & + & - & - \\
\hline
\end{tabular}

(1) Histological classification was based on the Japanese Gastric Cancer Classification guidelines [16]. Pap, papillary adenocarcinoma; tub1, well-differentiated tubular adenocarcinoma. ${ }^{(2)}$ Mixed, MUC5AC+/MUC2+; gastric, MUC5AC+/MUC2-. (3) These parameters were determined by examining 1000 epithelial cells and recording the frequency of positive nuclear staining.

\subsection{GHP Atypia and Expression of Cancer-Associated Proteins}

We next examined atypia in GHP samples and the expression of cancer-associated proteins, namely caudal type homeobox transcription factor 2 (CDX2), p53, and nucleostemin (NS) (Figure 3, Table 2). CDX2, which is associated with the acquisition of the intestinal phenotype associated with $H$. pylori infection [17], did not correlate with atypia (similarly to the results for $\mathrm{H}$. pylori and intestinal metaplasia). By contrast, p53 expression was negative in non-atypical GHPs and GHPs with low-grade atypia, but positive for $43 \%$ of high-grade GHP samples and $100 \%$ positive for the HPAGC samples. In addition, the number of cells that were positive for NS (a stem cell marker) increased in correlation with atypia.

\subsection{Features of HPAGC}

Finally, we examined the five cases in which cancer was found in the polyps (Table 3). In these cases, the size of the polyp exceeded $1 \mathrm{~cm}$ in four of the five cases. All cancer lesions were $4 \mathrm{~mm}$ or less intramucosal cancers with a well-differentiated histology and were carcinomas in situ, except for one case. Two cases exhibited CDX2-positive staining in $\geq 10 \%$ of the cells. One case had CDX2-positive cells in $5 \%$ of the cells, whereas the other two were negative for CDX2. Each of the three CDX2-positive cases was also characterized by a mixed mucin phenotype, whereas the two CDX2-negative cases exhibited a gastric mucin phenotype. There was no correlation between polyp size and p53 or NS indices, histology, CDX2 expression, or mucin type; and all of the cases were negative for KRAS G12D/G13D mutations. By contrast, BRAF V600E mutation was found in the two cases which exhibited a gastric mucin phenotype (Figure 4).

$B R A F$ exon 15 was sequenced in a low-grade atypia polyp and hyperplastic polypassociated gastric cancer (cases 2 and 3). In the low-grade atypia polyp, codon 600 exhibited the normal sequence, G-T-G (Val). By contrast, in the cancer cases, codon 600 was determined to be mutated, with the sequence G-T/A-G (Val/Glu). 

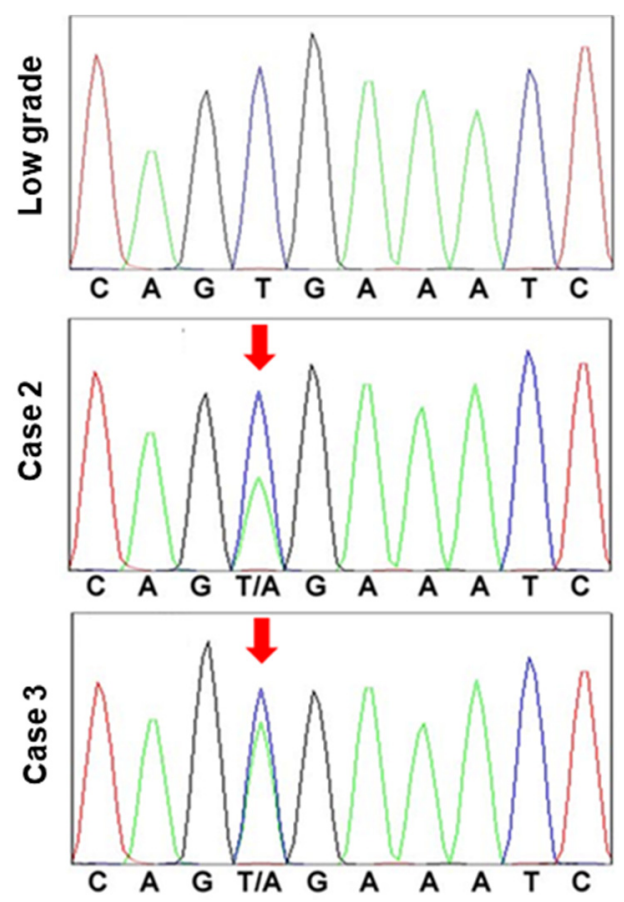

Figure 4. BRAF mutation in hyperplastic polyp carcinoma.

\section{Discussion}

In the present study, we analyzed the samples from 102 patients with GHPs. Intramucosal cancer was detected in five GHP cases (4.9\%). In these HPAGCs, CDX2-positive cells were found in three cases that also presented with mixed mucin phenotype, which is considered to be related to $H$. pylori infection. By contrast, two cases were CDX2-negative and exhibited a gastric mucin phenotype, and there was no evidence of $H$. pylori infection in the tumor or the surrounding mucosa. Although all of the HPAGC cases demonstrated p53 protein accumulation and high expression of a stem cell marker, it was particularly interesting that $B R A F$ mutations were identified in two of the five cases.

H. pylori and type A gastritis are associated with $89 \%$ of GHPs [18], and it is believed that GHPs develop during the repair of damaged mucosa [9]. The eradication of $H$. pylori has been reported to reduce the incidence of GHPs [19]. However, even if H. pylori infection is demonstrated in the mucosa of the non-polyp region, $\mathrm{H}$. pylori is not detected in the GHPs in one-third of the patients [20]. It was reported that the H. pylori infection rate in patients with GHPs (excluding polypoid foveolar hyperplasia and gastric mucosal prolapse polyps) was $21 \%$ [21]. This incidence is approximately equivalent to our data. In addition, although the H. pylori infection rate has declined in recent years, the prevalence of GHPs has increased, raising questions about $H$. pylori infection as the cause of GHPs [8]. CDX2 is a transcription factor that causes intestinal differentiation and it is expressed during gastric carcinogenesis with $H$. pylori infection, resulting in the acquisition of an intestinal phenotype and an increase in stemness $[17,22,23]$. In this study, atypical GHP was not correlated with $H$. pylori infection in the atypical lesion and the surrounding mucosa, intestinal metaplasia, or CDX2 expression. These findings suggest that, unlike non-polypderived gastric cancer, H. pylori infection is unlikely to be the main cause of HPAGC.

In non-polyp-derived gastric cancer, $H$. pylori infection induces genetic and epigenetic abnormalities $[24,25]$ as a result of intracellular signal disruption and induction of genetic instability by the CagA protein [26] and the activation of oxidative stress [27]. By contrast, the results from the current study indicated that increased GHP atypia did not correlate with $\mathrm{H}$. pylori infection, whereas 8-OHdG levels in atypical cells and the amount of granulation tissue in polyps or 4-HNE levels in granulation tissue did correlate with increased atypia. Moreover, these markers of oxidative stress also correlated with each other; however, H. pylori infection did not correlate with 8-OHdG or 4-HNE levels. These 
results suggest that inflammatory granulation tissue may be a source of oxidative stress in GHP, promoting genetic abnormalities associated with carcinogenic pathways. The association between inflammatory granulation tissue and carcinogenesis has been demonstrated using a foreign body carcinogenesis model, in which the generation of oxidative stress in foreign body granulation tissue was shown to be a key factor in cancer development [28]. The factors responsible for the formation of inflammatory granulation tissue in GHP are unclear. There is a morphological similarity between GHPs and cap polyps in the large intestine $[29,30]$. Because cap polyps are caused by mechanical stimuli such as mucosal prolapse syndrome [31], mechanical stimuli associated with gastric motility might be involved in the formation of inflammatory granulation tissue in GHP.

Another characteristic finding in this study was the presence of a BRAF mutation in HPAGC. Of the five HPAGC cases, two possessed the BRAF V600E mutation and a gastric mucin phenotype. BRAF is a downstream effector of KRAS, and its prognostic value in colorectal cancer is widely accepted [32]. However, $B R A F$ mutations occur at a very low frequency in gastric cancer, with only $2.9 \%$ of screened cases exhibiting a $B R A F$ mutation [33]. Similarly, KRAS mutation frequency is also very low in advanced gastric cancer $(4.9 \%)$, even in a report showing a comparatively high frequency [34]. These results indicate that, unlike in colorectal cancer, the oncogenic activation of mitogen-activated protein kinases by driver mutations is uncommon in gastric cancer. By contrast, the phosphatidylinositol 3-kinase catalytic subunit alpha (PIK3CA) mutation (which also occurs at a low frequency) was present in $5.5 \%$ of the cases; moreover, it is known to be highly correlated with Epstein-Barr virus-related gastric cancer [14]. These findings suggest that minor carcinogenic drivers, such as $B R A F$ mutations, might correlate with uncommon oncogenic pathways in gastric cancer.

$B R A F$ mutations are more common in colorectal cancer cases with mismatch repair gene abnormalities, but not in gastric cancer [35]. In this study, the $B R A F$ mutation-positive cases exhibited a gastric mucin phenotype. In serrated colorectal cancer, $B R A F$ mutations are known to correlate with CDX2 inactivation, leading to the expression of the gastric phenotype [36]. It is possible that the BRAF mutation in HPAGC might counteract the expression of the intestinal phenotype.

The p53 mutation is common in HPAGC $[37,38]$ and is thought to result in decreased expression of p21 (WAF1/CIP1; CDKN1a) and activation of cyclin D1 [39]. In the current study, abnormal accumulation of p53 protein was observed in all five cases of HPAGC. According to the subtype classification of gastric cancer due to molecular abnormalities as proposed by TCGA, many p53 mutations are found in the chromosomal instability subtype, which often exhibits an intestinal phenotype [14].

In this study, the HPAGC cases demonstrated a gastric phenotype and mutations of p53 and BRAF, as well as a possible association with chronic inflammation caused by mechanical stimuli, but no association with $H$. pylori infection. These characteristics suggest that HPAGC characterized by molecular abnormalities might comprise a new subtype with a distinct oncogenic background, albeit a minor population. All polyps analyzed in this study were very early lesions. Although it has been reported that BRAF mutations are more common in advanced stages [40], few studies have focused on early stage cancer. Genome instability (such as changes in gene copy numbers, rearrangements, and mutations) promotes clonal evolution of cancer cells through the accumulation of driver abnormalities [41]. It cannot be ruled out that $B R A F$ mutations found in early stage cancer might be replaced by new, more powerful drivers as the cancer progresses.

In this study, we analyzed HPAGC cases with a focus on GHPs. Although the number of target cases was 102, which is not large compared to that of previous studies, five cases of HPAGC were found. The proportion of GHPs which were associated with cancer among our cases was similar to that reported in previous studies. It should be emphasized that $B R A F$ mutations were found in two of five cases. This is a high frequency compared to previous studies on non-GHP-associated gastric cancer, but the small population of five cases requires further accumulation of cases. This finding suggests that a rare $B R A F$ 
gene mutation might specifically accumulate in GHPs, even though they exhibit a low carcinogenic frequency. These findings provide new insight into HPAGC that is worth investigating in a larger cohort.

$B R A F$ mutations are associated with poor prognosis in metastatic colorectal cancer [42], but treatment with drugs such as vemurafenib, which targets $B R A F$ mutations, has demonstrated high efficacy in melanoma patients [43]. The results of this study reaffirm the significance of $B R A F$ mutations as oncogenic drivers in gastric cancer and highlight the need to investigate HPAGC in a large number of GHP cases to determine the clinical significance of our findings.

\section{Materials and Methods}

\subsection{Patients}

We analyzed a total of 102 patients with GHPs who were subjected to endoscopic resection at the Miyoshi Central Hospital and histopathologically diagnosed by the Department of Molecular Pathology, Nara Medical University, Japan, between 2013 and 2019. As written informed consent was not obtained from the patients for their participation in the present study, all identifying information was removed from patient samples prior to their analysis to ensure strict privacy protection (unlinked anonymization). All procedures were performed in accordance with the Ethical Guidelines for Human Genome/Gene Research enacted by the Japanese Government and with the approval of the Ethics Committee of Nara Medical University, Japan (approval number, 937, 20 October 2014).

The endoscopic examination was performed by the two co-authors (TM and $\mathrm{KN}$ ) using a GIF-H260 or GIF-H260Z endoscope (Olympus Optical Co., Ltd., Tokyo, Japan) under white light observation, contrasted chromoendoscopy, and narrow band imaging.

\subsection{Histological Analyses}

Histological evaluation was performed using hematoxylin and eosin staining. Histological atypia was evaluated as follows: low grade, mild to moderate nuclear swelling; high grade, moderate to marked nuclear swelling, nuclear pseudostratification, and weakened mucus production; and cancer, structural atypia, and/or invasion, in addition to alterations similar to high-grade lesions. H. pylori infection and intestinal metaplasia were evaluated according to the updated Sydney classification [15]. Each grade (none, mild, moderate, and severe) was quantified as $0,1,2$, and 3 , respectively, and statistically analyzed. PAS staining grades $(0,0.5,1,2$, and 3$)$ were classified by the ratio of the area of the PAS-positive region in the cells: $0,0 \% ; 0.5,<10 \% ; 1,10-25 \% ; 2,25-50 \%$; and 3, $>50 \%$. Granulation tissue grade was evaluated by the area occupied by the polyp: $0,<10 \% ; 1,10-50 \% ; 2,50-75 \%$; and $3,>75 \%$.

\subsection{Immunohistochemistry}

Consecutive $4 \mu \mathrm{m}$ thick sections were immunohistochemically stained using the immunoperoxidase technique described previously [44], with the listed primary antibodies (Table 4) and appropriate secondary antibodies (Medical and Biological Laboratories, Nagoya, Japan) (all $0.2 \mu \mathrm{g} / \mathrm{mL}$ ). The tissue sections were then color-developed with diamine benzidine hydrochloride (Dako, Glostrup, Denmark) and counterstained with Meyer's hematoxylin (Sigma-Aldrich Chemical Co., St. Louis, MO, USA). Staining indices were evaluated by examining 1000 epithelial cells, and the frequency of positive nuclear staining was determined. For p53 and CDX2, an index of more than $10 \%$ was considered to represent a "positive case". 
Table 4. Antibodies used for immunohistochemistry.

\begin{tabular}{|c|c|c|c|c|}
\hline Target ${ }^{(1)}$ & Manufacturer ${ }^{(2)}$ & Clone & Code & $\begin{array}{c}\text { Working } \\
\text { Concentration }(\mu \mathrm{g} / \mathrm{mL})\end{array}$ \\
\hline $\mathrm{Ki}-67$ & DAKO-Agilent & MIB-1 & M7240 & 0.5 \\
\hline 8-OHdG & JaIKA & N45.1 & MOG-100P & 5 \\
\hline CDX2 & Abcam & CDX2-88 & ab157524 & 0.2 \\
\hline p53 & Abcam & PAb240 & ab26 & 1 \\
\hline NS & Abcam & - & ab70346 & 0.5 \\
\hline MUC5AC & DAKO-Agilent & CLH2 & M731601 & 0.5 \\
\hline MUC2 & DAKO-Agilent & Cср58 & M7313 & 0.5 \\
\hline
\end{tabular}

(1) 8-OHdG, 8-hydroxy-2'-deoxyguanosine; CDX2, caudal type homeobox transcription factor 2; NS, nucleostemin (2) DAKO-Agilent, Santa-Clara, CA, USA; JaIKA, Fukuroi, Japan; Abcam, Cambridge, UK.

\subsection{Enzyme-Linked Immunosorbent Assay (ELISA)}

In samples of polyp specimens, granulation tissues were manually dissected under a microscope using sterile needles from four consecutive $8 \mu \mathrm{m}$ thick sections of formalinfixed and paraffin-embedded blocks. For deparaffinization, $1 \mathrm{~mL}$ of xylene was added to the cells in $1.5 \mathrm{~mL}$ microcentrifuge tubes and mixed well using a vortex mixer for at least

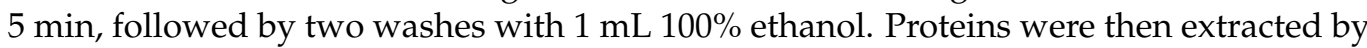
lysis with a RIPA buffer containing $0.1 \%$ SDS (Thermo Fisher, Tokyo, Japan). An ELISA kit (Abcam, Cambridge, MA, USA) was used to measure the concentration of 4-HNE. The assay was performed according to the manufacturer's instructions, and whole-cell lysates were used for the measurements.

\subsection{Mutation Analysis}

Five consecutive $8 \mu \mathrm{m}$ thick sections from formalin-fixed and paraffin-embedded blocks of polyp tumor specimens were analyzed for the presence of gene mutations in $B R A F$ exon 15 and KRAS codons 12 and 13. The samples were sent to SRL (Tokyo, Japan) for mutation screening, which was performed using the Luminex (xMAP) assay [45].

\subsection{Statistical Analyses}

Statistical significance was calculated using a two-tailed Fisher's exact test and ordinary ANOVA, using the InStat software (GraphPad, Los Angeles, CA, USA). Regression analysis was performed using the Pearson's regression test. Statistical significance was set at a two-sided $p$ value $<0.05$.

Author Contributions: R.F.-T. carried out most of the experiments and wrote the manuscript; A.O., H.K. (Hiroyuki Katsuragawa), S.K., S.M., T.S., K.F. and Y.N. assisted in the histopathological analysis; H.O. and C.N. assisted in the statistical analyses; Y.H., I.K. and T.M (Takuya Mori). assisted in the analysis and interpretation of results; K.N. and T.M (Takeshi Mizumoto). provided materials; H.K. (Hiroki Kuniyasu) and Y.L. conceived the project and designed the experiment; H.K. (Hiroki Kuniyasu) conceived the study concept. All authors have read and agreed to the published version of the manuscript.

Funding: This work was supported by MEXT KAKENHI Grant Numbers 19K16564 (R.F.-T.), 20K21659 (Hiroki Kuniyasu), 20K18007 (S.K.), 20K19349 (I.K.), 19K19915 (K.G.), 20K11260 (H.O.), 18K10788 (K.F.), the Natural Science Foundation of Jiangsu Education Department Project (17KJB320010) (Y.L.), and the National Natural Science Foundation of China (81702723) (Y.L.).

Institutional Review Board Statement: All procedures were performed in accordance with the Ethical Guidelines for Human Genome/Gene Research enacted by the Japanese Government and with the approval of the Ethics Committee of Nara Medical University, Japan (approval number, 937, 20 October 2014).

Informed Consent Statement: As written informed consent was not obtained from the patients for their participation in the present study, all identifying information was removed from patient samples prior to their analysis to ensure strict privacy protection (unlinked anonymization). 
Data Availability Statement: Not applicable.

Acknowledgments: The authors thank Tomomi Masutani for their expert assistance with the preparation of this manuscript.

Conflicts of Interest: The authors declare that the research was conducted in the absence of any commercial or financial relationships that could be construed as a potential conflict of interest.

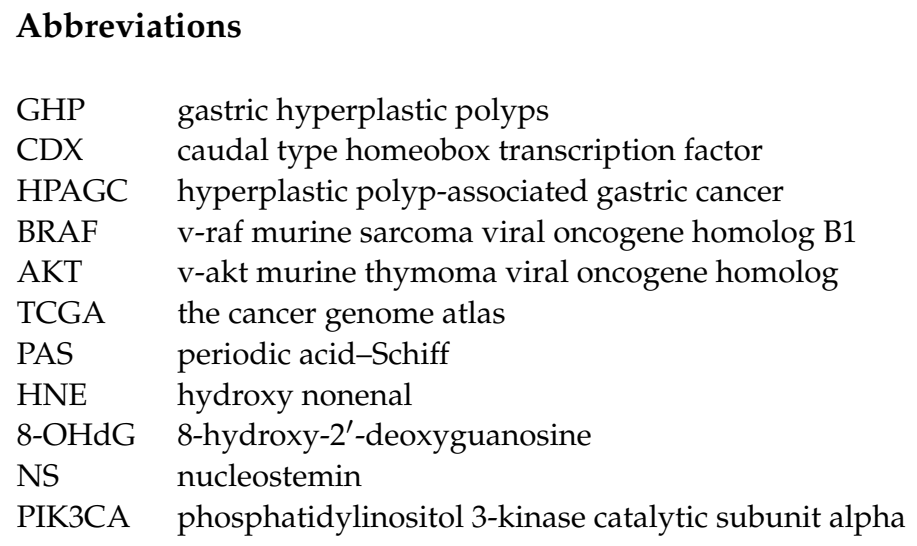

\section{References}

1. Statistics Bureau Ministry of Internal Affairs and Communications Japan. Statistical Handbook of Japan 2017; Statistics Bureau Ministry of Internal Affairs and Communications Japan: Tokyo, Japan, 2017.

2. Murata, M. Inflammation and cancer. Environ. Health Prev. Med. 2018, 23, 50. [CrossRef]

3. Watari, J.; Chen, N.; Amenta, P.S.; Fukui, H.; Oshima, T.; Tomita, T.; Miwa, H.; Lim, K.J.; Das, K.M. Helicobacter pylori associated chronic gastritis, clinical syndromes, precancerous lesions, and pathogenesis of gastric cancer development. World J. Gastroenterol. 2014, 20, 5461-5473. [CrossRef] [PubMed]

4. Sasaki, T.; Kuniyasu, H.; Luo, Y.; Kitayoshi, M.; Tanabe, E.; Kato, D.; Shinya, S.; Fujii, K.; Ohmori, H.; Yamashita, Y. Increased phosphorylation of AKT in high-risk gastric mucosa. Anticancer Res. 2013, 33, 3295-3300.

5. Kuniyasu, H.; Kitadai, Y.; Mieno, H.; Yasui, W. Helicobactor pylori infection is closely associated with telomere reduction in gastric mucosa. Oncology 2003, 65, 275-282. [CrossRef]

6. Kuniyasu, H.; Yasui, W.; Yokozaki, H.; Tahara, E. Helicobacter pylori infection and carcinogenesis of the stomach. Langenbeck Arch 2000, 385, 69-74. [CrossRef]

7. Tahara, E. Genetic pathways of two types of gastric cancer. IARC Sci. Pub. 2004, 157, 327-349.

8. Yu, X.; Wang, Z.; Wang, L.; Meng, X.; Zhou, C.; Xin, Y.; Sun, W.; Dong, Q. Gastric hyperplastic polyps inversely associated with current Helicobacter pylori infection. Exp. Ther. Med. 2020, 19, 3143-3149. [CrossRef] [PubMed]

9. Jain, R.; Chetty, R. Gastric hyperplastic polyps: A review. Dig. Dis. Sci. 2009, 54, 1839-1846. [CrossRef]

10. Takeuchi, C.; Yamamichi, N.; Shimamoto, T.; Takahashi, Y.; Mitsushima, T.; Koike, K. Gastric polyps diagnosed by double-contrast upper gastrointestinal barium $\mathrm{X}$-ray radiography mostly arise from the Helicobacter pylori-negative stomach with low risk of gastric cancer in Japan. Gastric Cancer 2017, 20, 314-321. [CrossRef] [PubMed]

11. Kushima, R.; Hattori, T. Histogenesis and characteristics of gastric-type adenocarcinomas in the stomach. J. Cancer Res. Clin. Oncol. 1993, 120, 103-111. [CrossRef]

12. Daibo, M.; Itabashi, M.; Hirota, T. Malignant transformation of gastric hyperplastic polyps. Am. J. Gastroenterol. 1987, 82, 1016-1025. [PubMed]

13. Orlowska, J.; Jarosz, D.; Pachlewski, J.; Butruk, E. Malignant transformation of benign epithelial gastric polyps. Am. J. Gastroenterol. 1995, 90, 2152-2159.

14. Cancer Genome Atlas Research Network. Comprehensive molecular characterization of gastric adenocarcinoma. Nature 2014, 513, 202-209. [CrossRef] [PubMed]

15. Dixon, M.F.; Genta, R.M.; Yardley, J.H.; Correa, P. Classification and grading of gastritis. The updated Sydney System. International Workshop on the Histopathology of Gastritis, Houston 1994. Am. J. Surg. Pathol. 1996, 20, 1161-1181. [CrossRef]

16. Japanese Gastric Cancer Association. Japanese classification of gastric carcinoma: 3rd English edition. Gastric Cancer 2011, 14, 101-112. [CrossRef]

17. Bornschein, J.; Malfertheiner, P. Helicobacter pylori and gastric cancer. Dig. Dis. 2014, 32, 249-264. [CrossRef]

18. Dirschmid, K.; Platz-Baudin, C.; Stolte, M. Why is the hyperplastic polyp a marker for the precancerous condition of the gastric mucosa? Virchows Arch. 2006, 448, 80-84. [CrossRef] 
19. Nam, S.Y.; Lee, S.W.; Jeon, S.W.; Kwon, Y.H.; Lee, H.S. Helicobacter pylori Eradication Regressed Gastric Hyperplastic Polyp: A Randomized Controlled Trial. Dig. Dis. Sci. 2020, 65, 3652-3659. [CrossRef] [PubMed]

20. Horvath, B.; Pai, R.K. Prevalence of Helicobacter pylori in Gastric Hyperplastic Polyps. Int. J. Surg. Pathol. 2016, 24, 704-708. [CrossRef]

21. Gonzalez-Obeso, E.; Fujita, H.; Deshpande, V.; Ogawa, F.; Lisovsky, M.; Genevay, M.; Grzyb, K.; Brugge, W.; Lennerz, J.K.; Shimizu, M.; et al. Gastric hyperplastic polyps: A heterogeneous clinicopathologic group including a distinct subset best categorized as mucosal prolapse polyp. Am. J. Surg. Pathol. 2011, 35, 670-677. [CrossRef]

22. Camilo, V.; Barros, R.; Sousa, S.; Magalhães, A.M.; Lopes, T.; Mário Santos, A.; Pereira, T.; Figueiredo, C.; David, L.; Almeida, R. Helicobacter pylori and the BMP pathway regulate CDX2 and SOX2 expression in gastric cells. Carcinogenesis 2012, 33, 1985-1992. [CrossRef]

23. Akhavan-Niaki, H.; Samadani, A.A. Molecular insight in gastric cancer induction: An overview of cancer stemness genes. Cell Biochem. Biophys. 2014, 68, 463-473. [CrossRef]

24. Hatakeyama, M. Helicobacter pylori and gastric carcinogenesis. J. Gastroenterol. 2009, 44, 239-248. [CrossRef] [PubMed]

25. Hamilton, J.P.; Meltzer, S.J. A review of the genomics of gastric cancer. Clin. Gastroenterol. Hepatol. 2006, 4, 416-425. [CrossRef]

26. Hatakeyama, M. Helicobacter pylori CagA and gastric cancer: A paradigm for hit-and-run carcinogenesis. Cell Host Microbe 2014, 15, 306-316. [CrossRef]

27. Butcher, L.D.; den Hartog, G.; Ernst, P.B.; Crowe, S.E. Oxidative Stress Resulting From Helicobacter pylori Infection Contributes to Gastric Carcinogenesis. Cell Mol. Gastroenterol. Hepatol. 2017, 3, 316-322. [CrossRef]

28. Okada, F. Beyond foreign-body-induced carcinogenesis: Impact of reactive oxygen species derived from inflammatory cells in tumorigenic conversion and tumor progression. Int. J. Cancer 2007, 121, 2364-2372. [CrossRef] [PubMed]

29. Campbell, A.P.; Cobb, C.A.; Chapman, R.W.; Kettlewell, M.; Hoang, P.; Haot, B.J.; Jewell, D.P. Cap polyposis-an unusual cause of diarrhoea. Gut 1993, 34, 562-564. [CrossRef] [PubMed]

30. Williams, G.T.; Bussey, H.J.R.; Morson, B.C. Inflammatory 'cap' polyps of the large intestine. Br. J. Surg. 1985, 72, S133.

31. Chetty, R.; Bhathal, P.S.; Slavin, J.L. Prolapse-induced inflammatory polyps of the colorectum and anal transitional zone. Histopathology 1993, 23, 63-67. [CrossRef]

32. Van Cutsem, E.; Köhne, C.H.; Láng, I.; Folprecht, G.; Nowacki, M.P.; Cascinu, S.; Shchepotin, I.; Maurel, J.; Cunningham, D.; Tejpar, S.; et al. Cetuximab plus irinotecan, fluorouracil, and leucovorin as first-line treatment for metastatic colorectal cancer: Updated analysis of overall survival according to tumor KRAS and BRAF mutation status. J. Clin. Oncol. 2011, 29, 2011-2019. [CrossRef] [PubMed]

33. Yang, Q.; Huo, S.; Sui, Y.; Du, Z.; Zhao, H.; Liu, Y.; Li, W.; Wan, X.; Liu, T.; Zhang, G. Mutation Status and Immunohistochemical Correlation of KRAS, NRAS, and BRAF in 260 Chinese Colorectal and Gastric Cancers. Front. Oncol. 2018, 8, 487. [CrossRef] [PubMed]

34. Takahashi, N.; Yamada, Y.; Taniguchi, H.; Fukahori, M.; Sasaki, Y.; Shoji, H.; Honma, Y.; Iwasa, S.; Takashima, A.; Kato, K.; et al. Clinicopathological features and prognostic roles of KRAS, BRAF, PIK3CA and NRAS mutations in advanced gastric cancer. BMC Res. Notes 2014, 7, 271. [CrossRef] [PubMed]

35. Oliveira, C.; Pinto, M.; Duval, A.; Brennetot, C.; Domingo, E.; Espín, E.; Armengol, M.; Yamamoto, H.; Hamelin, R.; Seruca, R.; et al. BRAF mutations characterize colon but not gastric cancer with mismatch repair deficiency. Oncogene 2003, 22, 9192-9196. [CrossRef]

36. Sakamoto, N.; Feng, Y.; Stolfi, C.; Kurosu, Y.; Green, M.; Lin, J.; Green, M.E.; Sentani, K.; Yasui, W.; McMahon, M.; et al. BRAF(V600E) cooperates with CDX2 inactivation to promote serrated colorectal tumorigenesis. eLife 2017, 6, e20331. [CrossRef] [PubMed]

37. Takayama, Y.; Ono, Y.; Mizukami, Y.; Itoh, H.; Nakajima, N.; Arai, H.; Tanaka, S.; Nobusawa, S.; Yokoo, H.; Onozato, Y. Comparative genome-wide analysis of gastric adenocarcinomas with hyperplastic polyp components. Virchows Arch. 2019, 475, 383-389. [CrossRef] [PubMed]

38. Kono, T.; Imai, Y.; Ichihara, T.; Miyagawa, K.; Kanemitsu, K.; Ajiki, T.; Kawasaki, K.; Kamigaki, T.; Ikuta, H.; Ohbayashi, C.; et al. Adenocarcinoma arising in gastric inverted hyperplastic polyp: A case report and review of the literature. Pathol. Res. Pract. 2007, 203, 53-56. [CrossRef]

39. Murakami, K.; Mitomi, H.; Yamashita, K.; Tanabe, S.; Saigenji, K.; Okayasu, I. p53, but not c-Ki-ras, mutation and down-regulation of p21WAF1/CIP1 and cyclin D1 are associated with malignant transformation in gastric hyperplastic polyps. Am. J. Clin. Pathol. 2001, 115, 224-234. [CrossRef]

40. Fu, X.H.; Chen, Z.T.; Wang, W.H.; Fan, X.J.; Huang, Y.; Wu, X.B.; Huang, J.L.; Wang, J.X.; Lin, H.J.; Tan, X.L.; et al. KRAS G12V Mutation is an Adverse Prognostic Factor of Chinese Gastric Cancer Patients. J. Cancer 2019, 10, 821-828. [CrossRef]

41. Pilié, P.G.; Tang, C.; Mills, G.B.; Yap, T.A. State-of-the-art strategies for targeting the DNA damage response in cancer. Nat. Rev. Clin. Oncol. 2019, 16, 81-104. [CrossRef]

42. Bokemeyer, C.; Van Cutsem, E.; Rougier, P.; Ciardiello, F.; Heeger, S.; Schlichting, M.; Celik, I.; Köhne, C.H. Addition of cetuximab to chemotherapy as first-line treatment for KRAS wild-type metastatic colorectal cancer: Pooled analysis of the CRYSTAL and OPUS randomised clinical trials. Eur. J. Cancer 2012, 48, 1466-1475. [CrossRef] 
43. Chapman, P.B.; Hauschild, A.; Robert, C.; Haanen, J.B.; Ascierto, P.; Larkin, J.; Dummer, R.; Garbe, C.; Testori, A.; Maio, M.; et al. Improved survival with vemurafenib in melanoma with BRAF V600E mutation. New Engl. J. Med. 2011, 364, 2507-2516. [CrossRef] [PubMed]

44. Kuniyasu, H.; Luo, Y.; Fujii, K.; Sasahira, T.; Moriwaka, Y.; Tatsumoto, N.; Sasaki, T.; Yamashita, Y.; Ohmori, H. CD10 enhances metastasis of colorectal cancer by abrogating the anti-tumoural effect of methionine-enkephalin in the liver. Gut 2010, 59, 348-356. [CrossRef] [PubMed]

45. Bando, H.; Yoshino, T.; Shinozaki, E.; Nishina, T.; Yamazaki, K.; Yamaguchi, K.; Yuki, S.; Kajiura, S.; Fujii, S.; Yamanaka, T.; et al. Simultaneous identification of 36 mutations in KRAS codons 61 and 146, BRAF, NRAS, and PIK3CA in a single reaction by multiplex assay kit. BMC Cancer 2013, 13, 405. [CrossRef] [PubMed] 